\title{
EchoGéo
}

$14 \mid 2010$

Afrique, 50 ans d'indépendance : Dynamiques spatiales, identités, circulations

\section{Contraintes climatiques dans les Préalpes françaises : évolution récente et conséquences potentielles futures}

Sylvain Bigot et Sandra Rome

\section{(2) OpenEdition}

Journals

Édition électronique

URL : https://journals.openedition.org/echogeo/12160

DOI : $10.4000 /$ echogeo.12160

ISSN : 1963-1197

Éditeur

Pôle de recherche pour l'organisation et la diffusion de l'information géographique (CNRS UMR 8586)

Référence électronique

Sylvain Bigot et Sandra Rome, «Contraintes climatiques dans les Préalpes françaises : évolution récente et conséquences potentielles futures », EchoGéo [En ligne], 14 | 2010, mis en ligne le 16 décembre 2010, consulté le 31 juillet 2021. URL : http://journals.openedition.org/echogeo/12160 ; DOI : https://doi.org/10.4000/echogeo.12160

Ce document a été généré automatiquement le 31 juillet 2021.

EchoGéo est mis à disposition selon les termes de la licence Creative Commons Attribution - Pas d'Utilisation Commerciale - Pas de Modification 4.0 International (CC BY-NC-ND) 


\title{
Contraintes climatiques dans les Préalpes françaises : évolution récente et conséquences potentielles futures
}

\author{
Sylvain Bigot et Sandra Rome
}

Les auteurs remercient Météo-France pour l'accès aux séries stationnelles (convention Climathèque) ou provenant de sorties de modèle, ainsi que la NOAA/OAR/ESRL/PSD (Boulder, Colorado) pour l'accès aux réanalyses atmosphériques NCEP; ce travail a été réalisé grâce aux soutiens de la DREAL Rhône-Alpes et de la Zone Atelier Alpes du CNRS.

\section{Introduction}

1 Dans le cadre de l'étude des impacts du changement climatique, plusieurs territoires possédant des enjeux environnementaux, économiques et sociétaux forts sont privilégiés. Il s'agit des milieux littoraux, des parcs naturels, des zones urbaines, des espaces agricoles et forestiers, ainsi que des territoires de montagne (MEEDDM, 2009). L'étude statistique de séries climatiques ou de données indirectes montre que les Alpes européennes ont déjà enregistré une hausse d'environ $2^{\circ} \mathrm{C}$ entre la fin du $19^{\mathrm{e}}$ et le début du $21^{\mathrm{e}}$ siècle, soit deux fois le taux de réchauffement mesuré à l'échelle nordhémisphérique (Auer et al., 2007). En conséquence, même une augmentation modérée des températures globales moyennes $\left(<2^{\circ} \mathrm{C}\right)$ dans les prochaines décennies, aura des conséquences socio-économiques et naturelles majeures, nécessitant des adaptations importantes (Bates et al., 2008). Les régions tempérées de moyenne montagne (i.e. entre 1000 et $2000 \mathrm{~m}$ pour l'exemple des Alpes françaises) deviennent alors l'espace potentiellement le plus vulnérable. Ainsi, les Préalpes françaises mêlent à la fois des enjeux économiques (urbanisation, industrie et tourisme), agricoles (élevage, pâturage et foresterie) et environnementaux (paysages et biodiversité). Cependant, la recherche sur la climatologie de cette région demeure limitée, avec beaucoup d'incertitudes sur 
les impacts locaux d'un futur changement climatique (Durand et al., 2009). Les incertitudes scientifiques proviennent pour une bonne part des imprécisions liées aux modèles climatiques régionaux (qui ne fournissent pas encore de prévisions régionales explicites, notamment pour les décideurs), mais aussi de l'absence d'un diagnostic pertinent obtenu à partir de séries longues provenant de l'observation. Pour l'interprétation des contraintes bioclimatiques, les Alpes françaises, réparties sur neuf départements, sont souvent découpées en aires simplifiées qui possèdent les mêmes critères bioclimatiques et écologiques (Ozenda, 1985). Mais cette cartographie statique, souvent ancienne, prend mal en compte la variabilité intra-saisonnière, essentielle en moyenne montagne, et pas du tout les variations interannuelles. Elle ne permet finalement pas de savoir quelle a été l'évolution contemporaine des principales contraintes climatiques, thermiques et pluviométriques, qui influencent l'ensemble des activités.

2 Ces différentes lacunes expliquent encore trop souvent le faible engagement politique pour préconiser des mesures d'adaptation anticipées et prévisionnelles aux prochaines variations climatiques en milieux de montagne (Agence Européenne pour l'Environnement, 2009). Elle peut aussi expliquer les approximations et terminologies abusives dès qu'on aborde ce sujet; citons l'exemple de la CIPRA (Commission Internationale pour la Protection des Alpes) qui écrit en 2009 que 'les Alpes doivent devenir une région modèle pour la protection du clima'!

3 En s'appuyant sur des séries climatiques analysées sur la période 1959-2009, cet article fait le point sur l'évolution des principaux paramètres climatiques à l'échelle des Préalpes du Nord (Vercors et Chartreuse, figure 1). Après avoir résumé les grands enjeux de l'étude du climat dans les espaces alpins (contraintes, impacts et adaptation), un diagnostic est proposé à partir de l'analyse statistique de séries stationnelles des deux massifs. Des données complémentaires (réanalyses atmosphériques et prévisions numériques régionales) sont ensuite utilisées pour préciser l'amplitude des différents changements observés et/ou attendus, ainsi que les possibles conséquences sur les principaux secteurs d'activités socio-économiques. 
Figure 1 - Localisation de l'espace étudié dans les Préalpes du Nord : les massifs du Vercors et de la Chartreuse

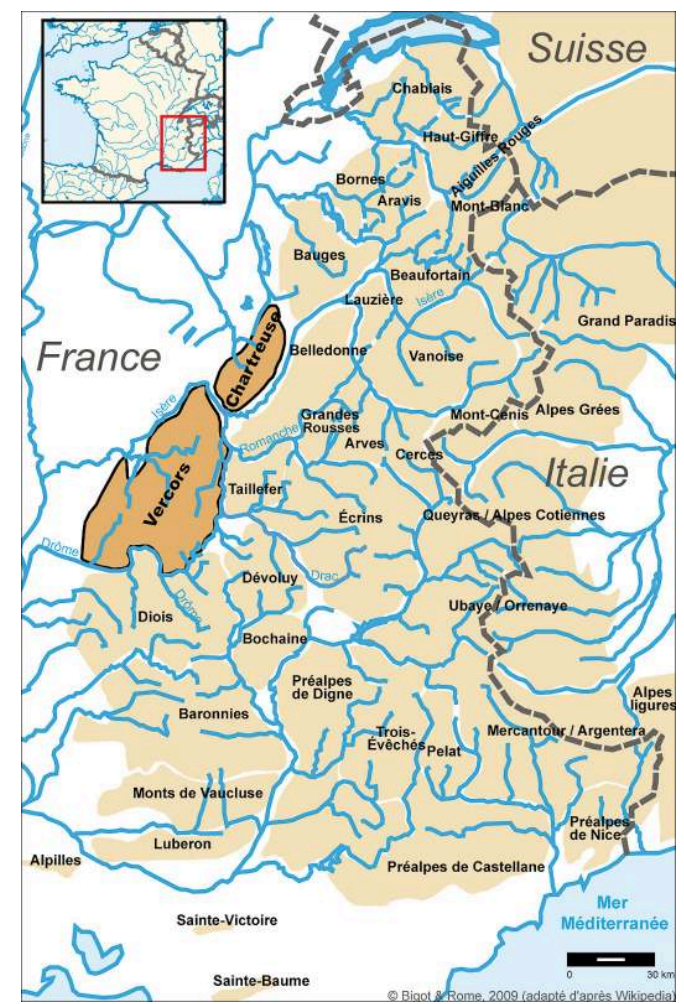

Source : S. Bigot et S. Rome, 2009 d'après un document de l'Encyclopédie Libre Wikipédia, 2008.

\section{Variabilité climatique et impacts potentiels sur les espaces de moyenne montagne}

Dans le cadre de la Convention Alpine, un plan d'action sur le changement climatique a été adopté en mars 2009, invitant les organisations, institutions et divers réseaux alpins à signer un 'Pacte sur le climat'. Celui-ci vise à faire des Alpes une région exemplaire dans la prévention et l'adaptation au changement climatique, avec un meilleur partage des informations sur le changement climatique alpin. Mais les disparités des situations locales et régionales sont tellement grandes qu'il est souvent difficile de comparer les domaines montagnards alpins. Les contrastes physiographiques favorisent les différences topoclimatiques et l'émergence de climats locaux (Beniston, 2006). Les stratégies d'adaptation devront donc avant tout être fonction des situations locales, solutions adéquates pour diminuer la vulnérabilité aux variations climatiques déjà observées ou attendues (figure 2). 
Figure 2 : Modèle conceptuel simplifié décrivant l'impact, la vulnérabilité et l'adaptation au changement climatique, en favorisant l'émergence de diagnostics régionaux

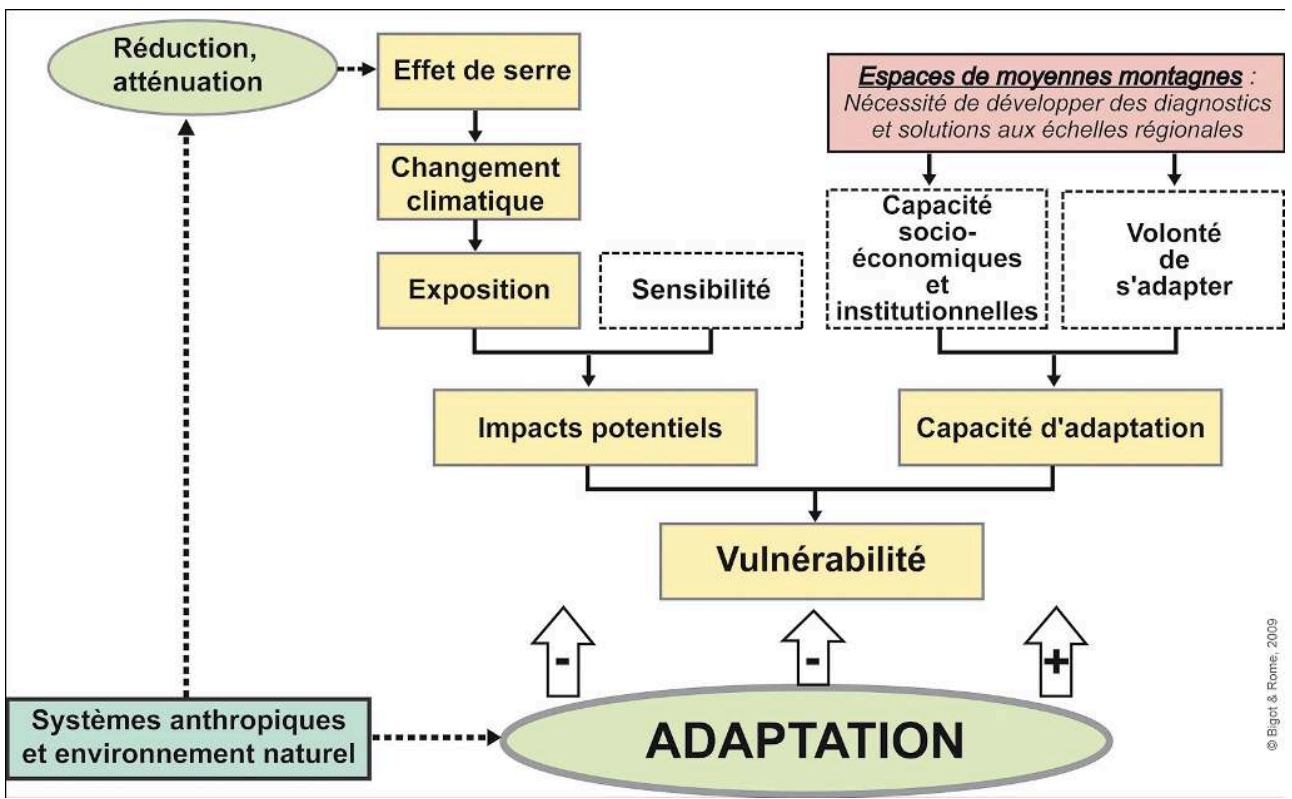

Source : S. Bigot et S. Rome, 2009 d'après Isoard et al., 2008 ; Agence Européenne pour l'Environnement, 2009

5 Les impacts du changement climatique ne seront ni uniformément, ni équitablement répartis à l'échelle des territoires de montagne, ces différences tenant autant à l'exposition aux aléas climatiques qu'aux spécificités géographiques et socioéconomiques territoriales susceptibles d'influencer la vulnérabilité des systèmes (MEEDDM, 2009). L'objectif scientifique est donc surtout de distinguer explicitement (avant de les faire reconnaître par les acteurs et gestionnaires) certains seuils critiques d'un point de vue écologique (en particulier les seuils d'irréversibilité et de nonlinéarité). Les principaux enjeux à étudier en moyenne montagne sont la ressource en eau, l'agriculture, la forêt et la biodiversité. La complexité vient en effet du fait que, sur le court ou moyen terme, le changement climatique (i.e. essentiellement le réchauffement atmosphérique et, dans une autre mesure, la modification des régimes pluviométriques) peut s'accompagner d'impacts positifs pour certaines activités (disponibilité en eau de fonte nivale et/ou glaciaire, augmentation de la croissance végétale, allongement du calendrier agricole, ...). Mais sur le moyen ou le long terme, il est alors essentiel de comprendre les possibles ruptures systémiques et renversement de tendance.

6 Les impacts écologiques potentiels du changement climatique en milieu de moyenne montagne peuvent se résumer à :

- une diminution des précipitations et une hausse de l'évapotranspiration (liée à

l'augmentation des températures) : une conséquence directe peut être la baisse de

l'écoulement lors des périodes estivales particulièrement sèches et/ou chaudes;

- un changement de temporalité de la pluviométrie et des débits, avec des séquences sèches plus longues et/ou plus fréquentes;

- un changement de l'enneigement (durée et hauteur de la couverture nivale, apparition et fusion nivale); 
- une baisse du niveau piézométrique avec, comme corollaire, l'augmentation des concentrations minérales des nappes ainsi que de la pollution d'origine anthropique, étant donné les temps de transfert plus longs au cours du printemps (sans compter l'élévation de la température hydrique) ;

- une élévation du pouvoir érosif de l'érosion hydrique ;

- une modification des écosystèmes dépendant des ressources en eau pluviales et/ou souterraines ;

- une transformation de la biocénose (phénologie, diversité et richesse spécifique, ...) ;

- une modification de la trame paysagère (rapport feuillus/conifères, qualité des pelouses, pousse de recrûs forestiers, ...).

7 Ces changements hydro- et bioclimatiques, avec leurs conséquences sur le biotope, affecteront l'ensemble des activités anthropiques préalpines, en particulier le tourisme (ANEM, 2007). La demande sociétale (élus, industriels, agriculteurs, gestionnaires, ...) est donc forte pour comprendre et anticiper les contrecoups des variations climatiques qui interagiront avec l'entretien et la mise en valeur des territoires préalpins. L'ensemble des filières agricoles est notamment dans l'attente de transformations significatives, touchant la qualité de l'alimentation, le maintien de l'élevage ovin, l'augmentation ou le maintien de la production, l'utilisation des différents parcours, les coûts et objectifs de production, ... (Gautier, 2004). La disparition (ou diminution) de la couverture nivale compromettra toute l'industrie du tourisme encore fondée, en moyenne montagne, sur les pratiques hivernales.

\section{La variabilité climatique des Préalpes du Nord (Vercors et Chartreuse)}

\section{Les chroniques climatiques étudiées sur la période 1959-2009}

Une recherche purement descriptive sur la climatologie alpine peut quelquefois donner l'impression d'un réseau stationnel important et spatialement cohérent, les Alpes européennes constituant d'ailleurs, à l'échelle mondiale, le meilleur réseau climatique in situ installé en zone de montagne (Beniston et al., 1997). Les travaux de synthèse menés sur le climat alpin relèvent cependant la faiblesse des longues séries et les incertitudes qui entourent leur homogénéité statistique (ONERC, 2008). Indépendamment des problèmes instrumentaux (déplacement, fermeture, modification du parc de mesure, absence des métadonnées décrivant les relevés), les difficultés les plus classiques proviennent surtout des spécificités de site liées à l'orographie et à la mesure des précipitations (forte anisotropie spatio-temporelle et mauvaise estimation des précipitations solides). A l'échelle des Alpes françaises, peu de travaux ont d'ailleurs cherché à combiner l'étude précise des séries nivales en relation avec d'autres paramètres climatiques.

9 A l'échelle alpine, Vidal et al. (2009) relèvent trois dates charnières dans les chroniques climatiques françaises: 1962-63 qui correspond à un faible nombre de relevés par rapport à la période précédente ; 1986-87 qui marque le début d'une augmentation significative des observations in situ; 1998-99 qui signale un palier atteint dans le nombre d'observations stationnelles. Durand et al. (2009) ajoutent que les travaux climatologiques sur le territoire français se basent souvent sur les régions de basses altitudes, en privilégiant l'examen des températures atmosphériques et des 
précipitions. De nombreux travaux ont été menés à l'échelle des Alpes européennes, mais en se servant de quelques paramètres climatiques et sans faire émerger les spécificités des variations préalpines (Frei et Schär, 1998; Moisselin et al., 2002; Spagnoli et al., 2002 ; Schmidli et al., 2002 ; Auer et al., 2005 ; Casty et al., 2005). L'étude spécifique du domaine préalpin reste à détailler, alors qu'il couvre pourtant $15000 \mathrm{~km}^{2}$ (11 $500 \mathrm{~km}^{2}$ pour les Préalpes du Sud et $3500 \mathrm{~km}^{2}$ pour les Préalpes du Nord). A l'échelle de la région Rhône-Alpes, les Préalpes du Nord correspondent à une zone de moyenne montagne allant du Diois jusqu'au Chablais, domaine surtout compris en moyenne dans la strate altitudinale 1000-2 $000 \mathrm{~m}$ (figure 3a). Elles se caractérisent par la diversité des climats régionaux, allant d'un climat tempéré océanique atténué à l'ouest, à un climat méditerranéen au sud, en passant évidemment par un climat montagnard et/ou tempéré à tendance continentale sur les parties centrales et orientales des Préalpes.

L'examen de 220 séries stationnelles (84 en Isère et 136 dans la Drôme) fournies par Météo-France indiquent que seules 31 sont utilisables lors d'une étude postérieure à 1955, avec une très forte anisotropie régionale (Bigot et al., 2007). En cherchant à retenir plusieurs paramètres météorologiques pour résumer les principales contraintes climatiques et bioclimatiques régionales (essentiellement pluviothermiques), le nombre de stations diminue encore fortement. Finalement, après études des séries stationnelles et des métadonnées à l'échelle de l'espace d'étude préalpin, seules quatre stations peuvent être retenues (figure 3b), le critère essentiel étant la recherche de la plus longue période commune disponible (1959-2009) sans lacunes sérielles, pour les paramètres climatiques suivants: les cumuls pluviométriques (RR), les températures minimales (Tn) et maximales ( $\mathrm{Tx}$ ) ainsi que leurs extrema mensuels, et le nombre de jours de gel (NBJG). Il s'agit d'Autrans (1 $090 \mathrm{~m}$, Isère), Monestier-de-Clermont (800 m, Isère), Saint-Pierre-de-Chartreuse (945 $\mathrm{m}$, Isère) et Saint-Jean-en-Royans $(308 \mathrm{~m}$, Drôme). La station de Saint-Pierre-de-Chartreuse a subi un déplacement en janvier 1961 (passant de 977 à $945 \mathrm{~m}$ d'altitude). Tout en restant à la même altitude, les stations de Monestier-de-Clermont et de Saint-Jean-en-Royans ont également été déplacées de quelques kilomètres, respectivement en décembre 1962 et en janvier 1980 ; Saint-Jeanen-Royans est aussi passée d'une station de type 4 (poste climatologique) à un type 2 (station automatique en temps réel) à partir de novembre 1992, ces différentes modifications instrumentales ayant pu avoir des conséquences sur la stationnarité sérielle, mais qu'il demeure difficile d'estimer. 
Figure $3 a$ - Carte de localisation des espaces de moyennes montagnes (1000-2000 m) dans les Alpes du Nord

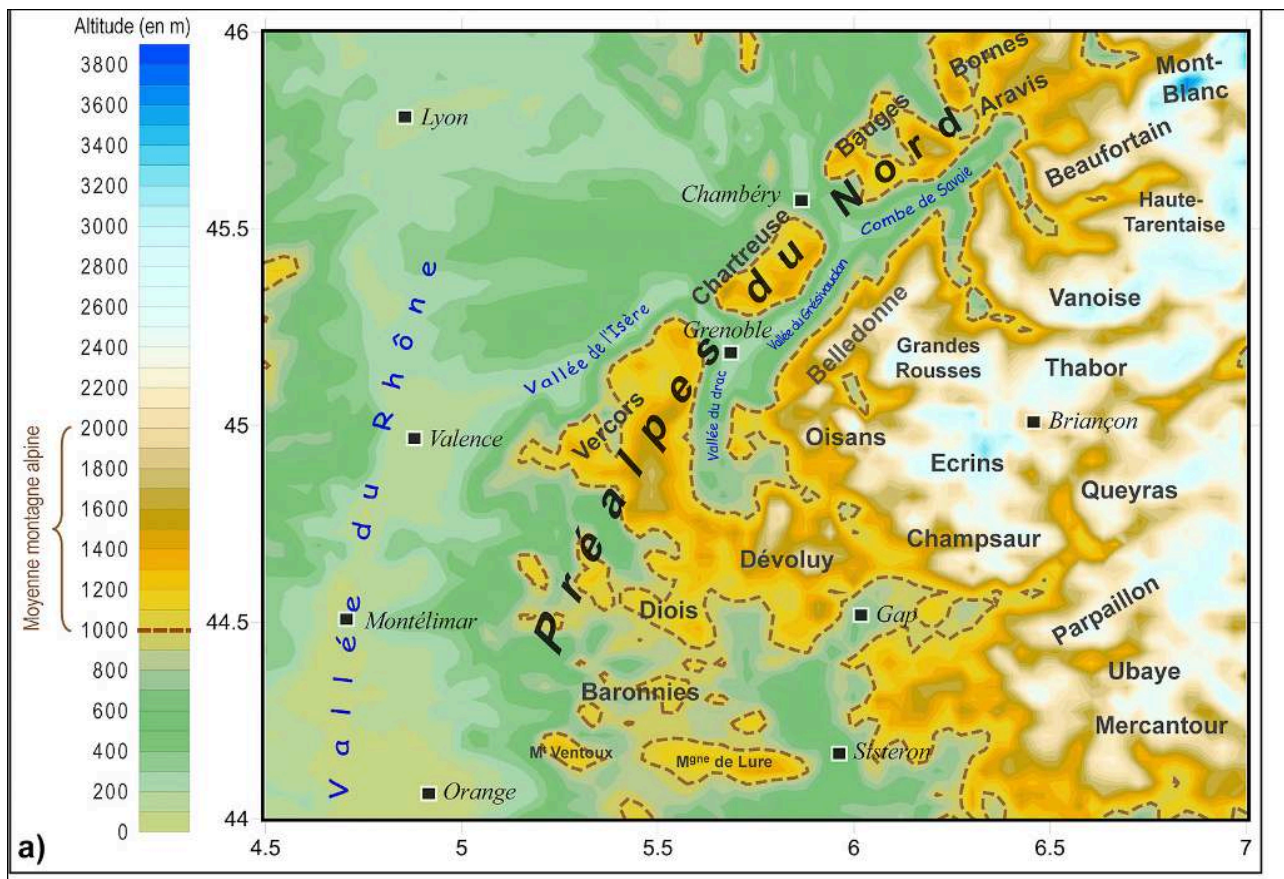

Utilisation du Modèle Numérique de Terrain ETOPO2, résolution de 2 minutes. Auteurs : S. Bigot et S. Rome, 2009. 
Figure $3 b$ - Localisation des quatre stations météorologiques retenues pour l'étude des variations climatiques sur la période 1959-2009 dans les massifs du Vercors et de la Chartreuse

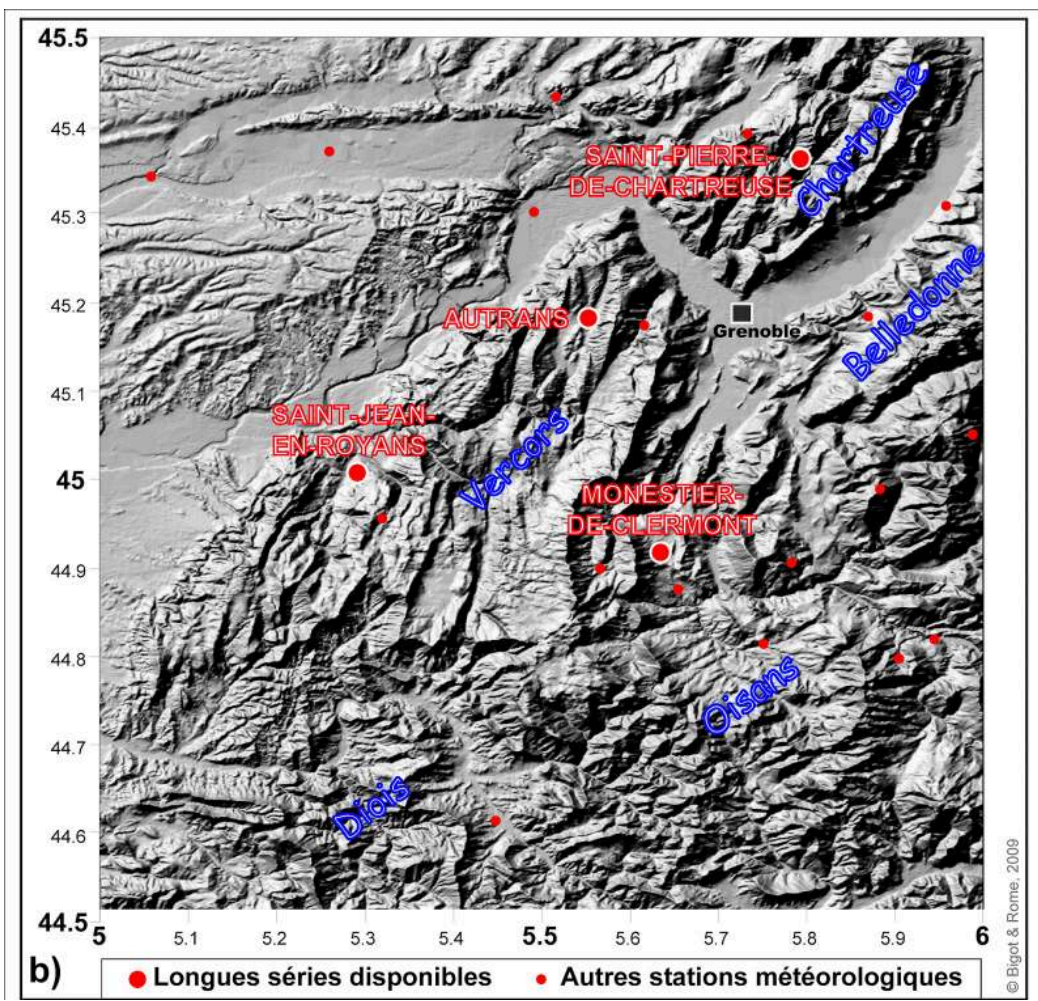

Utilisation d'un Modèle Numérique de Terrain de résolution de 75 m, fourni par le Parc Naturel Régional du Vercors.

Auteurs : S. Bigot et S. Rome, 2009

\section{Années extrêmes en fonction des précipitations et températures moyennes}

11 Un indice calculé à partir des 4 chroniques stationnelles révèle un climat régional moyen pluvieux (1417 mm/an) et tempéré $\left(8,8^{\circ} \mathrm{C}\right.$ de moyenne annuelle). Les totaux pluviométriques sont stables au cours de l'année (toujours supérieurs à $100 \mathrm{~mm} / \mathrm{mois}$ ), et les températures moyennes varient entre $0,7^{\circ} \mathrm{C}$ en janvier et $17,6^{\circ} \mathrm{C}$ en juillet. Parce que situées à une altitude moyenne de seulement $786 \mathrm{~m}$, ces températures stationnelles sont supérieures à celles citées par Durand et al. (2009) qui décrivent un climat alpin français à $1800 \mathrm{~m}$ aux températures comprises entre $3,4^{\circ} \mathrm{C}$ au nord (massif du Chablais) et $5,1^{\circ} \mathrm{C}$ au sud (massif du Mercantour). En terme de gradients climatiques verticaux, il apparaît que certains massifs intra-alpins comme Belledonne, le Beaufortain ou le Mont-Blanc possèdent plutôt des caractéristiques climatiques moyennes préalpines, tandis que le Vercors ressemble davantage aux massifs alpins centraux.

12 L'étude pluviothermique menée à partir des anomalies (valeurs centrées réduites à partir de la moyenne 1959-2009; figure 4) montre que sur les 51 années d'étude, 16 apparaissent extrêmes (i.e. avec une anomalie dépassant 1 en valeur absolue) en période hivernale (ici le bimestre janvier-février), et 13 en été (bimestre juillet-août). Cependant, alors que les extremums hivernaux sont enregistrés dans les quatre quadrants, indiquant l'existence de tous les types pluviothermiques possibles, seuls deux types apparaissent vraiment en été $i$ ) les années anormalement chaudes et 
sèches, comme 1983 ; ii) les années anormalement fraîches et pluvieuses, comme 1960. On observe que l'été 2003, exceptionnellement chaud, n'est en revanche pas considéré dans les années les moins pluvieuses de l'espace Chartreuse-Vercors. Cette différence de typologie des extrêmes entre l'hiver et l'été est surtout due àla plus grande complexité des situations météorologiques (donc des circulations et des combinaisons pluviothermiques) ainsi qu'à la plus grande variabilité interannuelle durant la période hivernale. Cependant, à l'échelle régionale, c'est bien durant l'été que l'on observe la plus grande variété de types de circulation atmosphérique associés à des extrêmes thermiques (Rome et al., 2009).

Figure 4a - Relation bivariée entre les anomalies thermiques et pluviométriques mensuelles calculées pour un indice préalpin (moyenne de 4 stations : Saint-Pierre-de-Chartreuse, Autrans, Saint-Jean-en-Royans et Monestier-de-Clermont) sur la période 1959-2009 pour la moyenne janvier-février

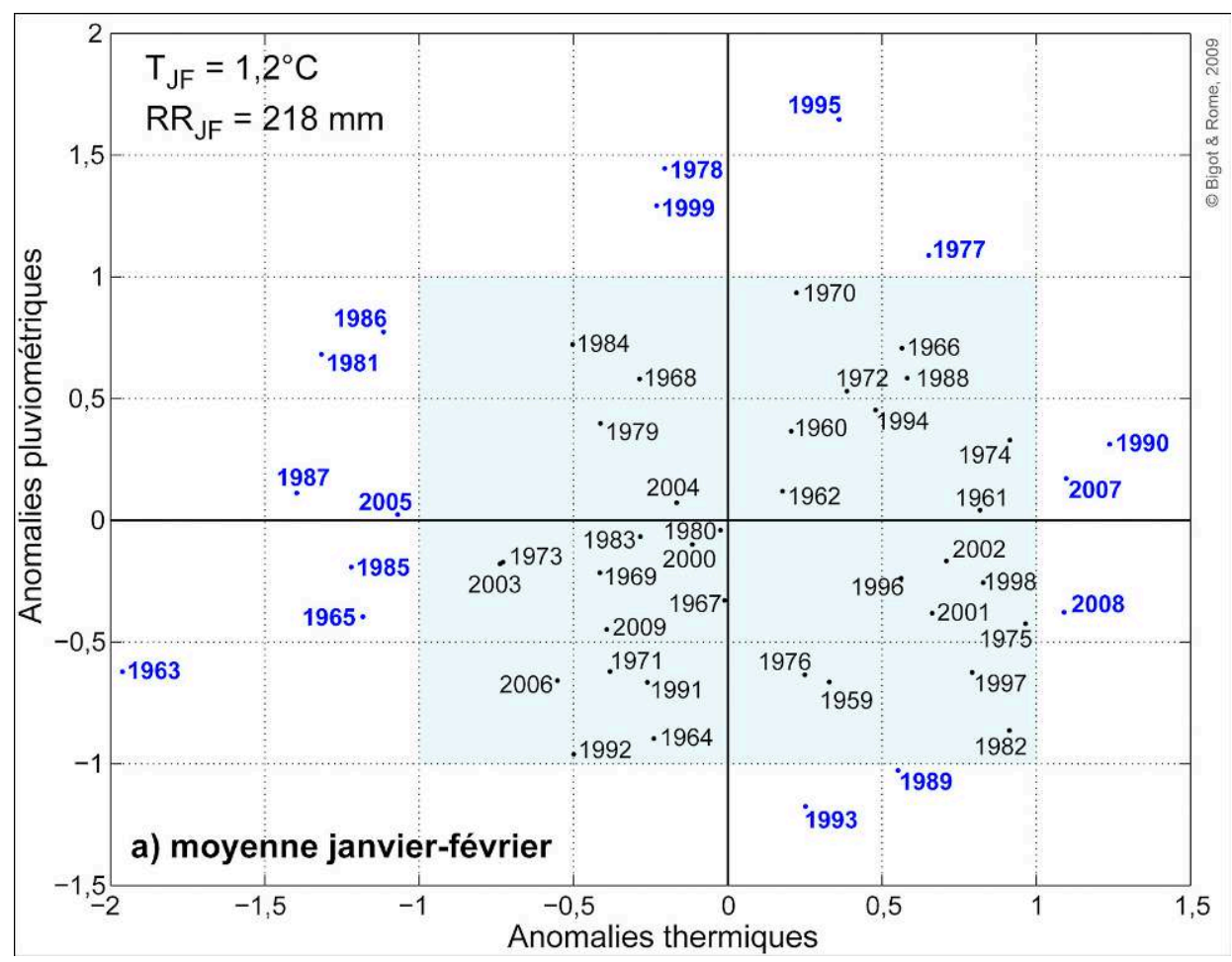

Auteurs : S. Bigot et S. Rome, 2009 
Figure $4 \mathrm{~b}$ - Relation bivariée entre les anomalies thermiques et pluviométriques mensuelles calculées pour un indice préalpin (moyenne de 4 stations : Saint-Pierre-de-Chartreuse, Autrans, Saint-Jean-en-Royans et Monestier-de-Clermont) sur la période 1959-2009 pour la moyenne juilletaoût

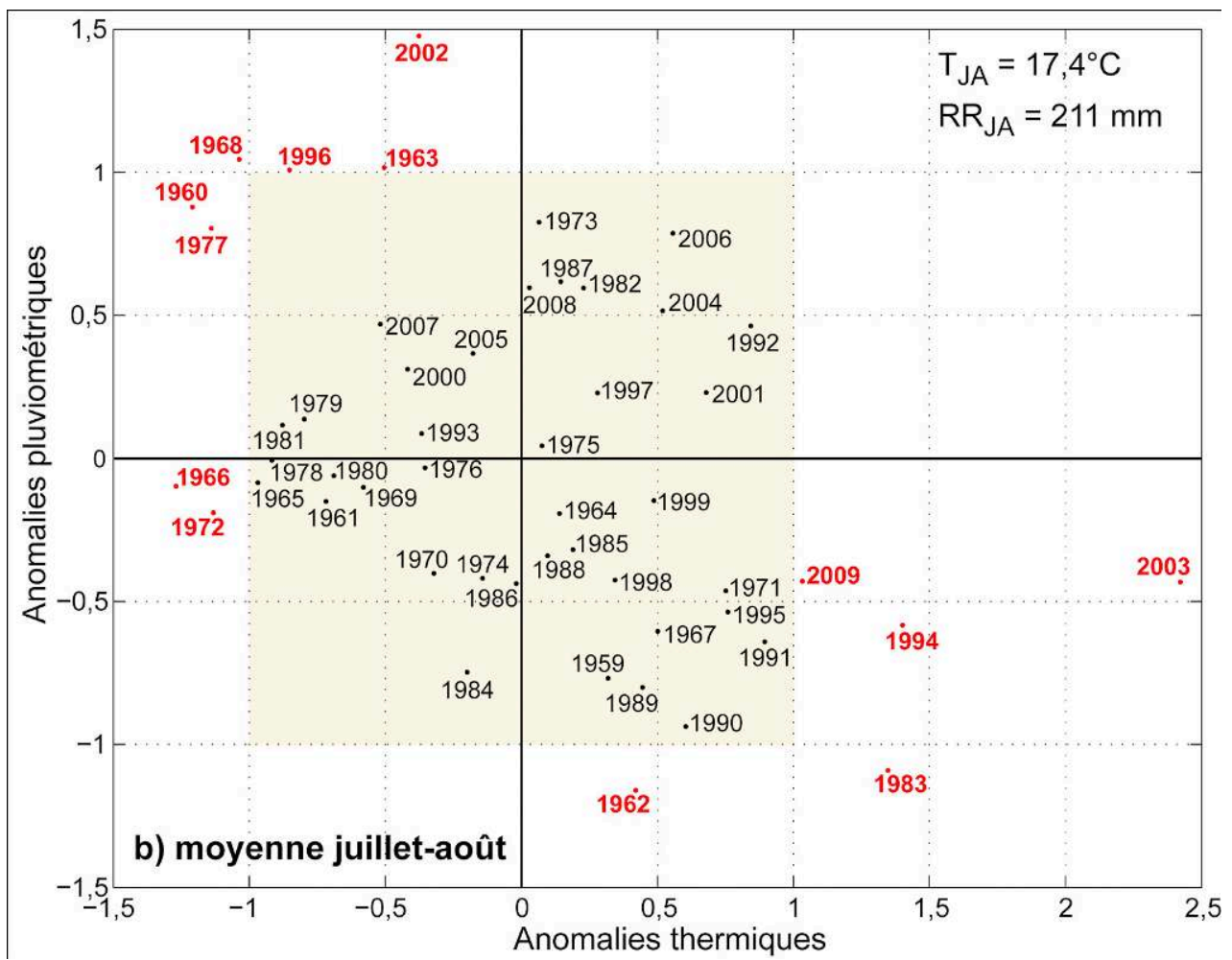

Auteurs: S. Bigot et S. Rome, 2009

Les anomalies correspondent aux écarts centrés réduits ; les anomalies les plus importantes, i.e. dépassant |1|, sont indiquées en bleu pour la période hivernale et en rouge pour la période estivale ; les valeurs thermiques (en ${ }^{\circ} \mathrm{C}$ ) et pluviométriques (en $\mathrm{mm}$ ) moyennes sont indiquées pour chacun des bimestres.

\section{Une évolution pluviothermique significative après 1988}

13 L'analyse de la variabilité interannuelle révèle surtout un fait marquant, commun à tous les principaux paramètres climatiques étudiés $\left(\mathrm{T}_{\text {moy }}, \mathrm{RR}, \mathrm{Tn}\right.$ et $\left.\mathrm{Tx}\right)$, soit une rupture sérielle datée vers 1988 (figure 5). Quoique significative pour les totaux pluviométriques (au seuil de $95 \%$ selon un test de Mann-Kendall), elle est surtout nette (au seuil de $99 \%$ ) pour les séries thermiques et correspond à une augmentation importante des températures atmosphériques régionales. En revanche, aucune tendance claire n'apparaît vraiment dans les cumuls pluviométriques annuels et saisonniers régionaux (Bigot et al., 2006). Cela peut d'abord s'expliquer par la grande variabilité spatio-temporelle des précipitations en milieu de montagne, étroitement dépendante des conditions topographiques locales. Grâce à une combinaison observation/modélisation, Durand et al. (2009) observent pourtant une tendance pluviométrique positive depuis 1958 dans les Alpes du Nord d'environ $12 \mathrm{~mm} / \mathrm{an}$, notamment pour les massifs situés entre 1500 et $2000 \mathrm{~m}$ (aucune tendance significative n'est détectée dans les Alpes du Sud).

La hausse régionale des températures peut être évaluée à environ $0,014^{\circ} \mathrm{C} / \mathrm{an}$ pour les températures moyennes, et affecte aussi bien la station la plus froide (Autrans) que 
celles au climat plus doux (Monestier-de-Clermont et Saint-Jean-en-Royans). A partir de 21 stations réparties sur toutes les Alpes françaises, Durand et al. (2009) estiment l'élévation des températures à environ $0,026^{\circ} \mathrm{C} / \mathrm{an}$, soit presque le double de ce qui est observé ici pour le domaine Vercors-Chartreuse.

Figure 5 - Variations climatiques interannuelles des écarts à la moyenne (1959-2009) de quatre stations des Préalpes du Nord (Autrans, $1090 \mathrm{~m}$; Monestier-de-Clermont, $800 \mathrm{~m}$; Saint-Jean-enRoyans, $308 \mathrm{~m}$; Saint-Pierre-de-Chartreuse, $945 \mathrm{~m}$ ) pour : a) les températures mensuelles moyennes $\left(\mathrm{en}^{\circ} \mathrm{C}\right)$; b) le cumul mensuel des précipitations (en $\left.\mathrm{mm}\right)$; c) les températures minimales mensuelles moyennes $\left(\mathrm{en}^{\circ} \mathrm{C}\right) ; \mathrm{d}$ ) les températures maximales mensuelles moyennes $\left(\mathrm{en}{ }^{\circ} \mathrm{C}\right)$

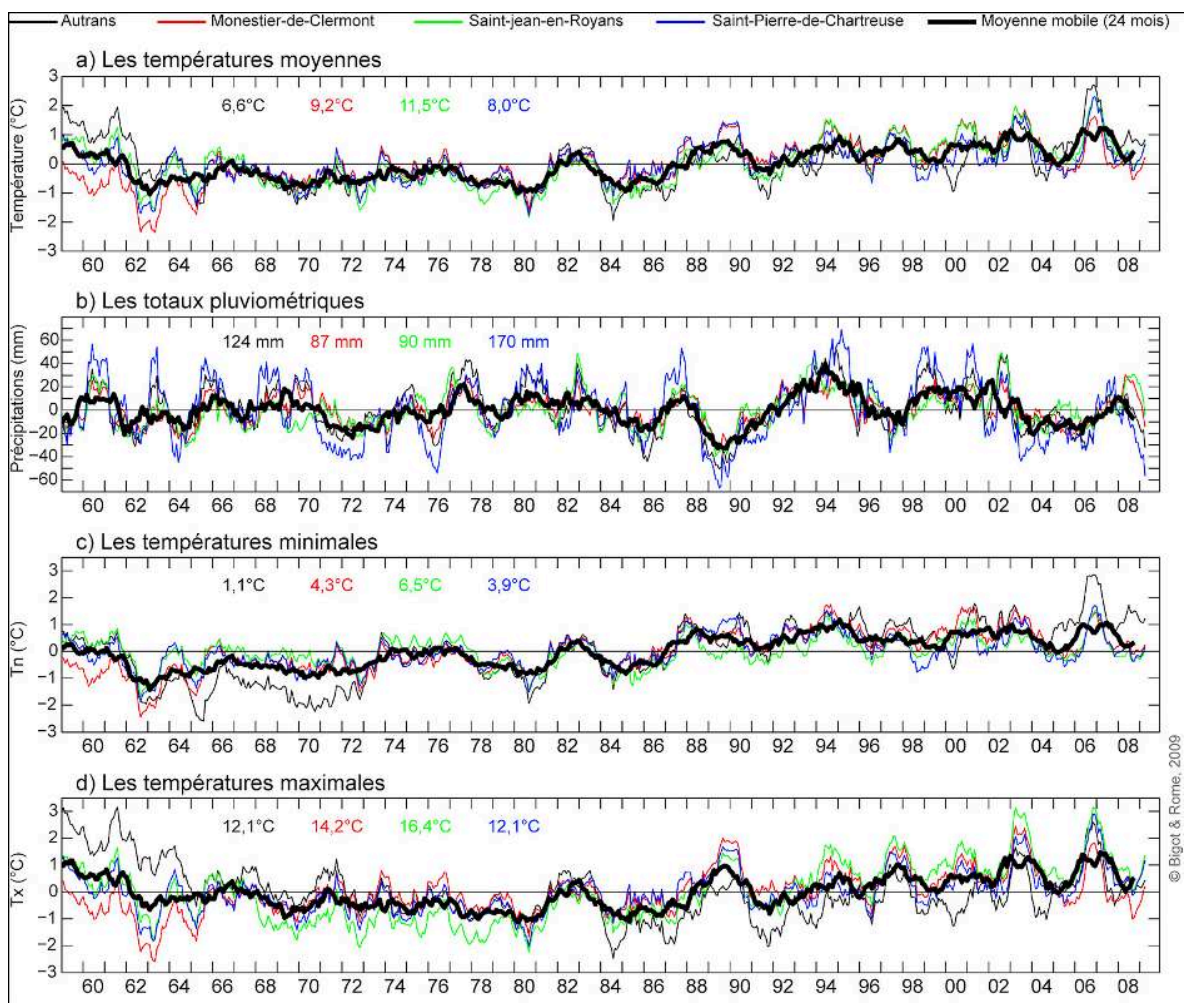

Les chroniques stationnelles mensuelles sont filtrées grâce à une moyenne mobile de 12 mois ; la courbe noire en gras représente la moyenne des quatre séries lissée sur une fenêtre de 24 mois ; les valeurs mensuelles moyennes sont indiquées pour chaque paramètre et pour chaque station ; les données brutes sont obtenues sur la période allant de janvier 1959 à août 2009 auprès de MétéoFrance.

Auteurs : S. Bigot et S. Rome, 2009

La tendance des températures minimales et maximales n'est pas toujours totalement synchrone et de même intensité. En fait, à l'échelle française, la hausse des températures minimales présente un gradient est-ouest, tandis que celle des maximales offre un gradient nord-sud (Moisselin et al., 2002). Une explication généralement avancée pour le gradient des tendances des minimales est l'accroissement de la nébulosité sur les façades océaniques. Le gradient méridien des Tx serait davantage lié à des processus dépendant à la fois du continent et du bassin méditerranéen, le climat $\mathrm{du}$ domaine vercorien étant particulièrement sensible à ce type de mécanismes (Bigot et al., 2006). L'examen plus précis des données météorologiques quotidiennes de la station d'Autrans permet de mieux juger du changement des températures. On observe en effet très clairement d'une part, que la fréquence des Tn comprise entre $-6^{\circ} \mathrm{Cet}+2^{\circ} \mathrm{C}$ diminue après 1987 tandis que celles supérieures à $+6^{\circ} \mathrm{C}$ augmentent, les modifications fréquentielles n'étant pas significatives à l'échelle des Tx (figure 6a) ; d'autre part, d'un 
point de vue saisonnier, cela se traduit surtout par une diminution de l'amplitude thermique quotidienne, particulièrement entre début mars et fin octobre (figure 6b). Plus généralement, d'un point de vue thermique, cela se traduit aussi par un printemps plus précoce et un hiver plus tardif. Comme conséquence immédiate en moyenne montagne, la durée moyenne de l'enneigement s'est réduite de plus d'un mois depuis la décennie 1970 et la hauteur moyenne de neige a diminué d'environ $40 \mathrm{~cm}$ dans les Alpes du Nord, alors qu'à haute altitude (>2500 m), l'impact sur la couverture neigeuse est négligeable (ONERC, 2008).

Figure 6a - Comparaison des températures minimales (Tn) et maximales (Tx) journalières à la station d'Autrans calculées sur les périodes 1959-1987 et 1988-2008 pour les fréquences thermiques

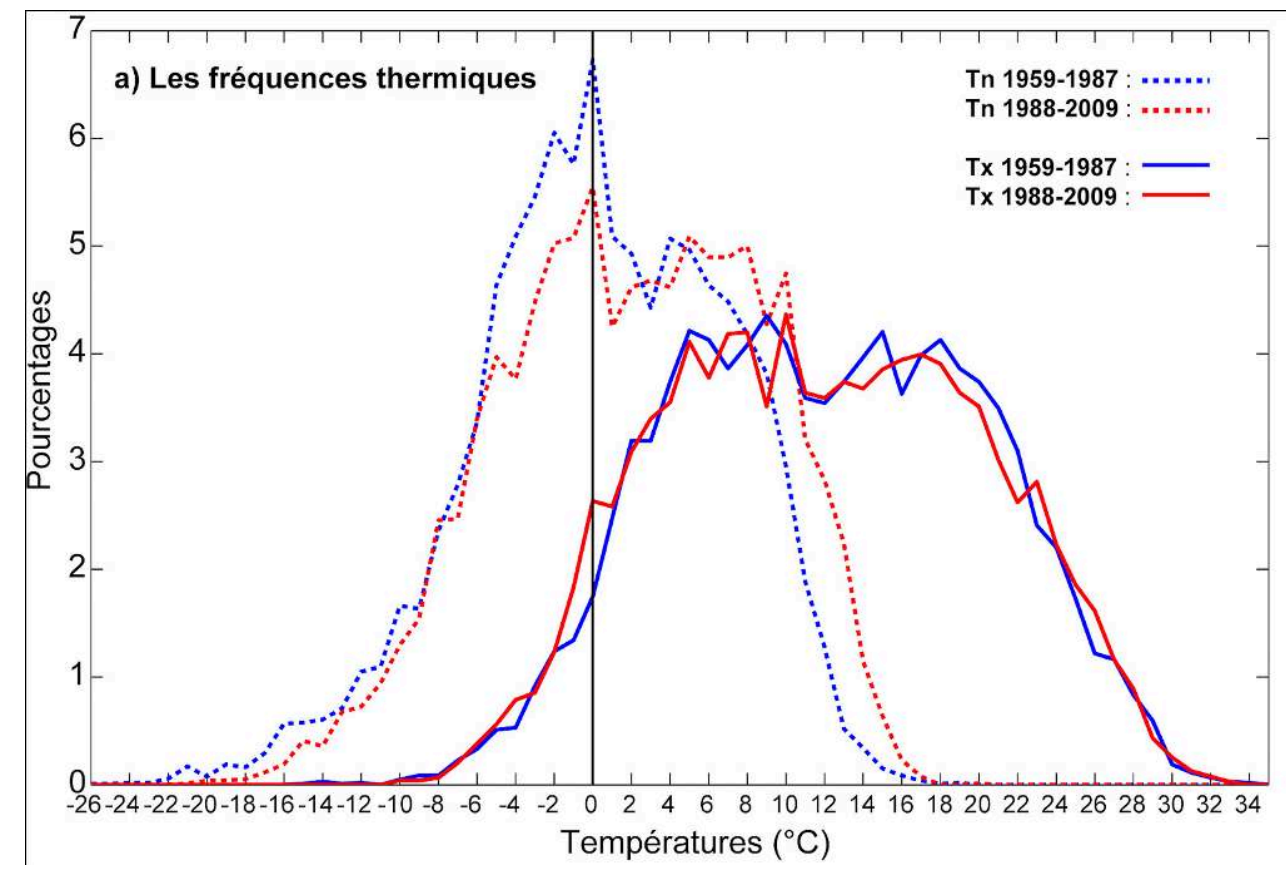

Auteurs : S. Bigot et S. Rome, 2009 ; données Météo-France. 
Figure $6 \mathrm{~b}$ - Comparaison des températures minimales $(\mathrm{Tn})$ et maximales $(\mathrm{Tx})$ journalières à la station d'Autrans calculées sur les périodes 1959-1987 et 1988-2008 pour les températures moyennes

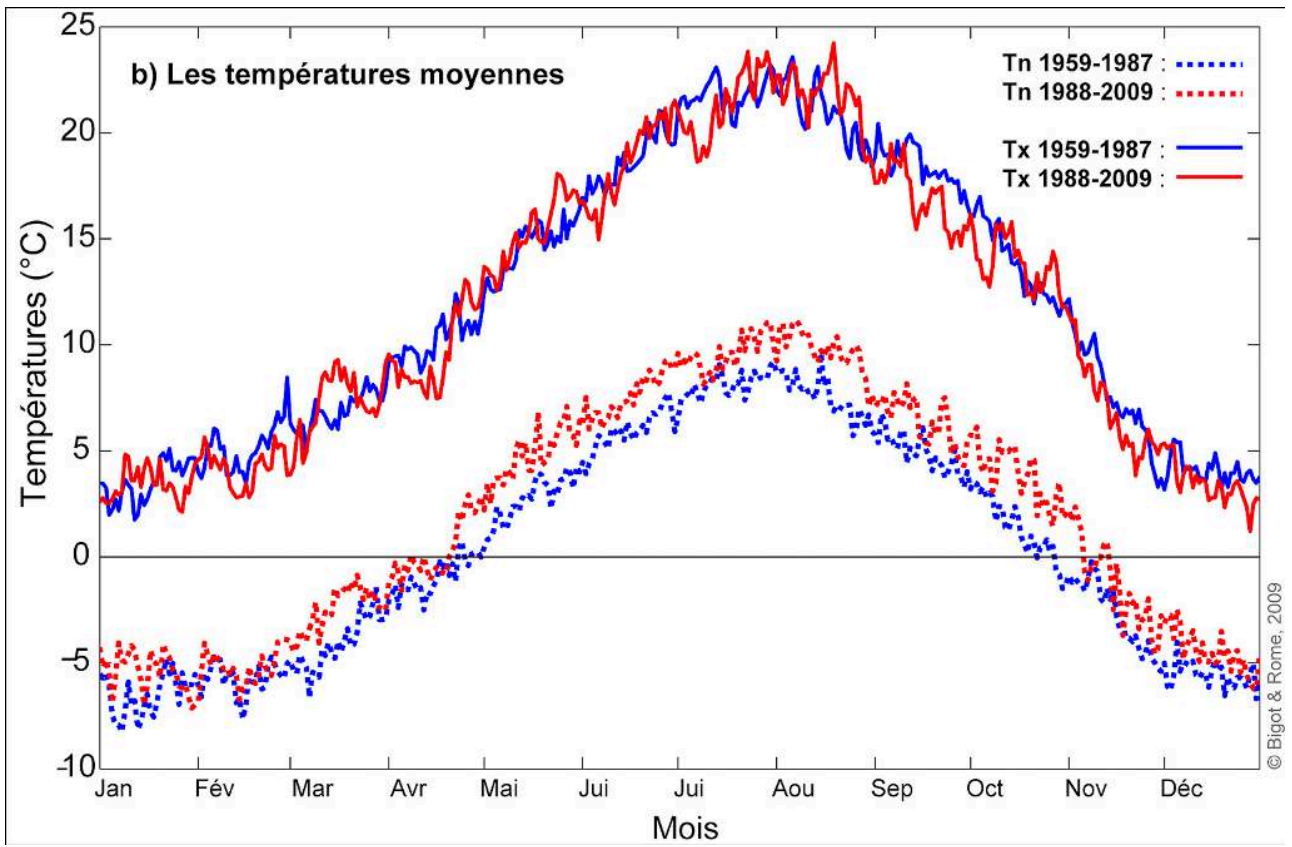

Auteurs : S. Bigot et S. Rome, 2009 ; données Météo-France.

L'étude de paramètres thermiques complémentaires permet d'affiner le diagnostic de ces variations. Ainsi, cette partie des Préalpes enregistre une baisse significative du nombre de jours de gel à partir de 1987-88, même si les années 1999 et 2004-05 se situent plutôt légèrement au-dessus de la moyenne cinquantennale (figure 7a). L'évolution des valeurs thermiques extrêmes (extremums des Tn et des Tx) montre que ce sont surtout les températures les plus froides $\left(\mathrm{Tn}_{\min }\right)$ qui augmentent brutalement après 1987 (figure 7b). Ces variations correspondent surtout à des hivers moins rigoureux avec, en particulier, une diminution significative du nombre de jours de gel diurne. Les températures les plus chaudes $\left(\mathrm{Tx}_{\max }\right)$ s'élèvent en revanche fortement à partir de 1997 (figure 7e), traduisant finalement une tendance thermique régionale composée de plusieurs phases. 
Figure 7 - Variations climatiques interannuelles des écarts à la moyenne (1959-2009) de quatre stations des Préalpes du Nord (Autrans, $1090 \mathrm{~m}$; Monestier-de-Clermont, $800 \mathrm{~m}$; Saint-Jean-enRoyans, $308 \mathrm{~m}$; Saint-Pierre-de-Chartreuse, $945 \mathrm{~m}$ ) pour : a) le nombre de jour de gel ; b) le minimum mensuel des températures minimales $\left(e{ }^{\circ} \mathrm{C}\right) ; \mathrm{c}$ ) le maximum mensuel des températures minimales $\left(e n{ }^{\circ} \mathrm{C}\right)$; d) le minimum mensuel des températures maximales (en $\left.{ }^{\circ} \mathrm{C}\right)$; e) le maximum mensuel des températures maximales $\left(e{ }^{\circ} \mathrm{C}\right)$

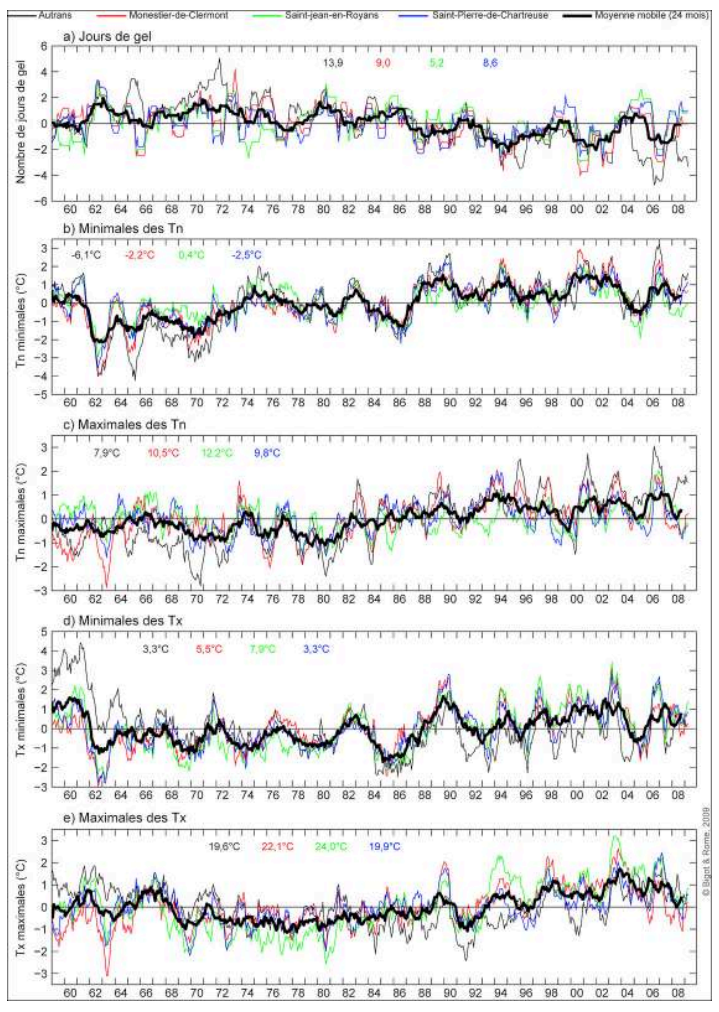

Auteurs : S. Bigot et S.Rome, 2009.

Ces résultats précisent ceux obtenus à l'échelle globale (Trenberth et al., 2007) ou alpine (Durand et al., 2009), indiquant une augmentation des températures depuis les années 1980, quelle que soit l'altitude considérée, ainsi qu'une baisse du nombre de jours de gel de 12 à $14 \%$ (ONERC, 2008). Parmi les causes de ce réchauffement atmosphérique régional, le déplacement vers le nord des systèmes de hautes pressions subtropicales de méso-échelle est souvent avancé. Le renforcement des situations de blocages anticycloniques sur l'Europe et/ou sur le domaine alpin est décrit par plusieurs auteurs (Beniston, 2006; Rome et al., 2009). Plusieurs d'entre eux notent que ces variations interannuelles peuvent être, en partie du moins, associées à l'Oscillations NordAtlantique (ONA). Les phases positives de l'ONA (renforcement des flux d'ouest sur l'Europe) se traduisent généralement par une diminution anormale des précipitations dans les Alpes (également associée à une augmentation des températures). Durand et al. (2009) observent une corrélation positive entre l'ONA et la température de l'air plus importante dans les Alpes du Nord que dans les Alpes du Sud. A l'échelle du domaine alpin Isère-Drôme, en utilisant 31 stations météorologiques, son influence reste relativement mineure (i.e. part de variance commune inférieure à $30 \%$ ) et chronologiquement très instable (Bigot et al., 2007).

L'augmentation des pressions atmosphériques a pour conséquence, surtout en milieu de montagne, l'allongement de la durée quotidienne et saisonnière de l'ensoleillement (Auer et al., 2007). De même, alors que la vitesse du vent synoptique mesuré à l'échelle 
européenne au cours du $20^{e}$ siècle diminue (Matulla et al., 2007), l'influence des contraintes topoclimatiques locales augmenterait, puisque directement dépendantes du flux radiatif incident (donc aussi de la nébulosité). Le poids des conditions géographiques locales (altitude, exposition, encaissement, écoulements, ...), relativement différentes pour les 4 stations étudiées, évolue donc. Cette complexité physiographique, quelquefois quasi stationnelle, demeure encore difficile à simuler précisément dans les modèles numériques du climat, même ceux développés à échelle régionale, par exemple le modèle SAFRAN à l'échelle des Alpes françaises (Durand et al., 2009). Cependant, lorsque l'on corrèle une série stationnelle étudiée avec le champs thermique européen (continental et océanique) provenant de réanalyses climatiques, on observe une contraction nette des corrélations positives autour de chaque station (i.e. son aire représentative) après 1988 (figure 8). Les variations thermiques interannuelles Vercors-Chartreuse sont maintenant davantage similaires à celles observées en France méditerranéenne qu'à celles du domaine intra-alpin.

Figure 8 - Corrélations linéaires calculées entre les températures de surface (niveau $995 \mathrm{hPa}$ ) issues des réanalyses atmosphériques NCEP/NCAR et la station d'Autrans (série obtenue auprès de Météo-France) sur deux périodes: a) 1959-1987, b) 1988-2008

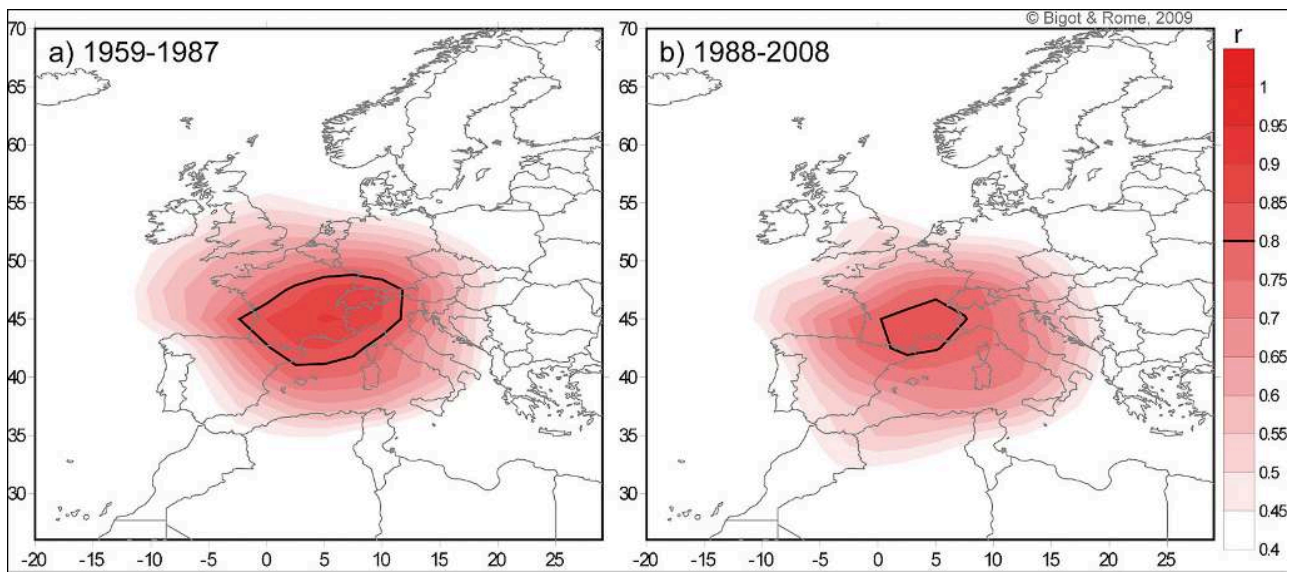

Seuls les coefficients de corrélation ( $r$ ) significatifs au seuil de $99 \%$ sont indiqués en dégradés de rouge ; l'isoligne noire indique le coefficient de corrélation 0,80 (soit environ $64 \%$ de variance commune

Auteurs : S. Bigot et S. Rome, 2009

\section{Quelles projections pluviothermiques régionales?}

Depuis quelques années seulement, les tentatives de modélisation numérique du climat se multiplient à l'échelle régionale, notamment en zone de montagne (Terray et Braconnot, 2007). Les résultats de différentes simulations provenant de plusieurs modèles européens prévoient que c'est en moyenne dans le domaine occidental des Alpes que les augmentations de températures (i.e. la variations entre $\mathrm{T}_{1971-2000}$ et $\mathrm{T}_{2071-2100}$ ) seront maximales, supérieures à $4,5^{\circ} \mathrm{C}$ (ONERC, 2008). L'élévation serait de l'ordre de $3^{\circ} \mathrm{C}$ à $5{ }^{\circ} \mathrm{C}$ en hiver, et plutôt de $4{ }^{\circ} \mathrm{C}$ à $6^{\circ} \mathrm{C}$ en été, c'est-à-dire au-dessus des valeurs prévues à l'échelle européenne. La plupart des simulations s'accordent pour suggérer que ce sont les espaces de moyennes montagnes qui enregistreront les hausses les plus significatives, surtout dans la seconde moitié du $21^{\mathrm{e}}$ siècle (Agence Européenne pour l'Environnement, 2009). Les cumuls pluviométriques changeront également, particulièrement à l'échelle saisonnière: les modèles prévoient une 
diminution des pluies estivales, mais une augmentation des précipitations printanières et hivernales. Ces dernières seront principalement reçues sous forme liquide, la durée de la couverture nivale se réduisant fortement. D'ici 2100 et à moins de $1500 \mathrm{~m}$ d'altitude, les chutes de neige pourraient diminuer de plus de $30 \%$ en hiver, la neige ayant quasiment disparu en-deçà de $500 \mathrm{~m}$ (Jacob et al., 2007). Ces changements conduiraient donc à des changements importants des bilans hydriques régionaux aux phases hivernale et estivale (écoulement de surface, infiltration, évapotranspiration, ...), mais qu'il reste extrêmement difficile de quantifier, surtout à l'échelle locale.

Grâce au modèle atmosphérique ARPEGE-Climat de Météo-France, des simulations ont été effectuées selon deux scénarios d'émissions de gaz à effet de serre, en fonction d'un ensemble d'hypothèses d'évolution de la population et de l'économie mondiales (Terray et Braconnot, 2007). Il s'agit de scénarios du Groupe d'experts Intergouvernemental sur l'Evolution du Climat (GIEC) à l'horizon 2100 par rapport à la période 1980-1999, où le scénario A2 (dit 'intensif) simule une augmentation des émissions de gaz à effet de serre proche de celle d'aujourd'hui (avec une hausse thermique de $+3,8^{\circ} \mathrm{C}$ en moyenne); le scénario B2 (dit 'modéré) modélise une augmentation moins rapide des émissions de gaz à effet de serre, avec une augmentation des températures de $+2,4^{\circ} \mathrm{C}$ en moyenne (GIEC, 2007). Pour ce type d'étude, Météo-France rappelle qu'il s'agit évidemment de résultats avec une marge d'incertitudes liées au modèle (global et pas régional) et aux principes physiques inhérents, mais que cela fournit cependant des estimations très plausibles du climat des prochaines décennies.

21 Même si la tendance globale des sorties de modèle est plus significative qu'une valeur ponctuelle des estimations, une comparaison est faite à l'échelle préalpine (i.e. celle du point de grille ARPEGE) entre la situation contemporaine, résumée grâce à la série d'Autrans, et la prévision valable pour 2050 (figure 9). Indépendamment de l'hypothèse de départ du modèle, le réchauffement hivernal et estival semble significatif à l'échelle régionale, puisque aucune température négative n'est reproduite, et que les valeurs thermiques dépassent régulièrement $25^{\circ} \mathrm{C}$ en juillet-août (soit une anomalie prévisionnelle supérieure à $5{ }^{\circ} \mathrm{C}$ ). En hiver, l'élévation prévue est surtout sensible pour les températures minimales $(\mathrm{Tn})$, conformément à ce qui a été observé depuis la fin des années 1980 , de l'ordre de $+4{ }^{\circ} \mathrm{C}$. 
Figure 9 - : Comparaison entre d'une part, les températures minimales ( $\mathrm{Tn})$ et maximales (Tx) hebdomadaires moyennes à la station d'Autrans calculées sur la période 1988-2008 (courbes noires) et, d'autre part, les estimations pour 2050 issues du modèle ARPEGE-Climat selon un scénario modéré (i.e. B2 du GIEC) et intensif(i.e. A2 du GIEC) pour la maille du modèle correspondant à la station

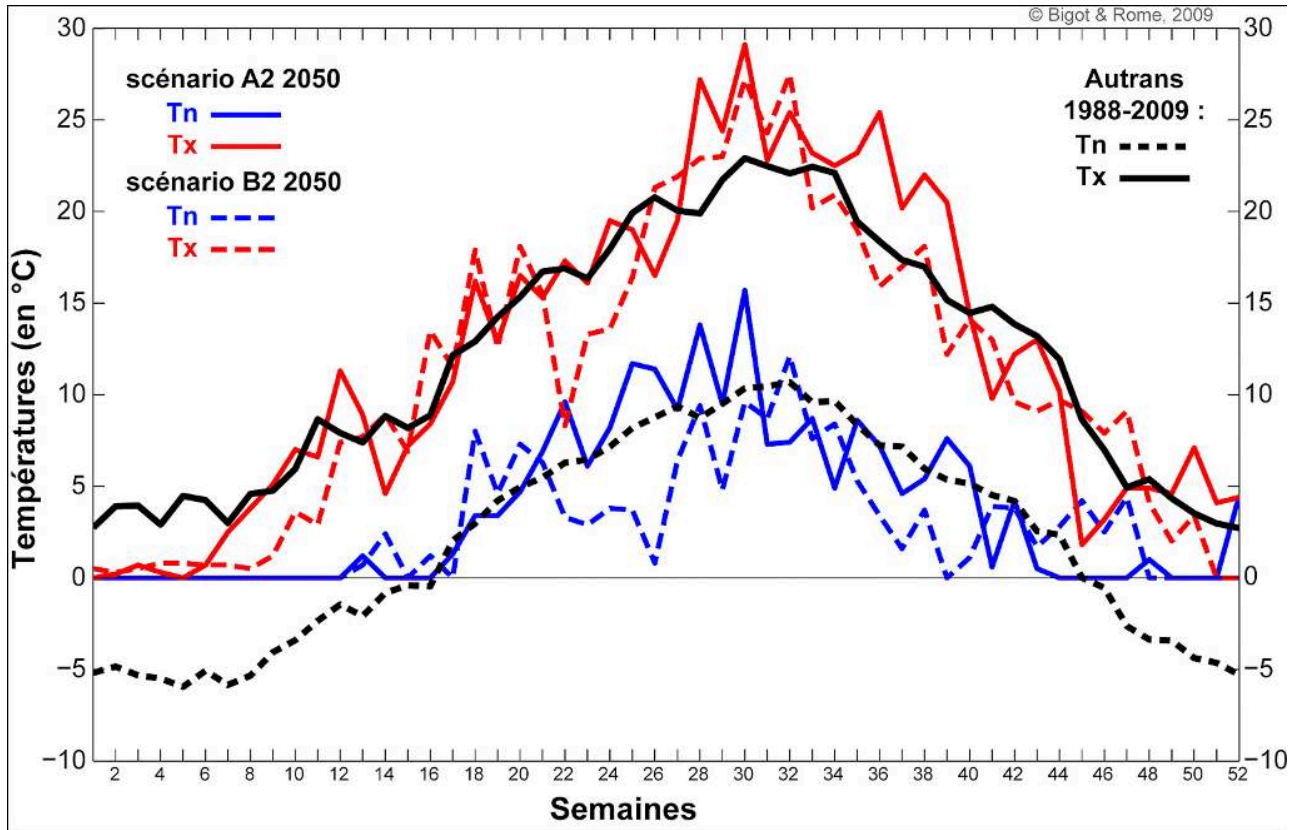

Auteurs : S. Bigot et S. Rome, 2009 ; données ARPEGE et stationnelles obtenues auprès de MétéoFrance

\section{Les conséquences possibles sur les principales activités socio-économiques}

En fonction de l'impact social et paysager de ces changements climatiques, ainsi que des investissements économiques considérables déjà réalisés en moyenne montagne, mais aussi des projets envisagés à court (2010-2015) ou moyen terme (2015-2050), deux secteurs d'activités sont surtout visés à l'échelle Vercors-Chartreuse : l'agriculture et le tourisme.

\section{Agrosystème et pratiques agricoles}

Les variations bioclimatiques déjà observées à l'échelle des Préalpes (ou celles attendues en fonction des simulations numériques) ont des impacts écologiques complexes (particulièrement phénologiques), donc des conséquences agronomiques à plus ou moins court terme sur les activités agricoles, pastorales ou forestières. Le réchauffement a notamment pour effet principal d'atténuer les contraintes climatiques liées à l'altitude. Ainsi, l'Association Nationale des Elus de Montagne note que les perspectives sur le secteur agricole de montagne sont a priori moins alarmistes que pour d'autres zones géographiques, puisque l'élévation des températures et de l'ensoleillement s'accompagne théoriquement d'une augmentation de la biomasse végétale et d'un allongement de la période végétative (ANEM, 2007). 

aussi des retombées immédiates sur les rendements et les coûts du travail, qui peuvent impliquer des baisses de productivité. Ainsi, le réchauffement hivernal (notamment des Tn) et la diminution du nombre de jours de gel observés depuis la fin des années 1980 modifie d'abord l'un des principaux forçages écologiques du cycle végétatif des espèces de montagne, c'est-à-dire la période de fusion nivale. Les études empiriques menées dans le Vercors et fondées sur des observations biogéographiques prévoient par exemple, sur la base d'une augmentation des températures atmosphériques moyennes de $1{ }^{\circ} \mathrm{C}$ d'ici 2030 , une forte diminution de la zone écologique dite 'de combat' (espace de transition entre étages alpin et sub-alpin) et sa quasi disparition d'ici 2100 si la température augmentait de $4{ }^{\circ} \mathrm{C}$, comme le prévoient les modèles (Bigot et Rome, 2009). A des altitudes inférieures $(<1000 \mathrm{~m})$ et dans des espaces topoclimatiques particuliers (par exemple, certaines vallées intérieures), les changements de régimes ombrothermiques pourraient augmenter le stress hydrique de la végétation à cause des épisodes secs et diminuer ainsi sa productivité. Cela risque alors d'accroître la vulnérabilité de certains espaces forestiers de moyenne montagne à différents facteurs de perturbation biotiques (scolytes, parasites) et abiotiques (vent, feu). Les changements d'amplitudes thermiques diurnes conjugués aux tendances pluviothermiques interannuelles transforment la saisonnalité et la qualité des espaces herbacés, avec une durée potentielle d'occupation des zones d'estive plus longues par les systèmes d'élevage. Traditionnellement, ces derniers se distinguent clairement par une phase hivernale se déroulant en stabulation et une autre phase estivale en alpage. Ainsi, pour la plupart des éleveurs, le début du printemps détermine la pousse prairiale qui, si elle est tardive, oblige à l'apport d'alimentation concentrée pour le bétail (Gautier, 2004). La chronologie de la séquence bioclimatique hivernale est également déterminante, puisqu'elle détermine le calendrier des gestations et les entrées en bergerie, donc, en conséquence, tout le travail d'astreinte de l'agriculteur (alimentation, gardiennage, surveillance à la mise bas, ...) ainsi que la main d'œuvre impliquée. L'alimentation fournie en hiver découle donc finalement des fourrages récoltés en été-automne, le rendement en herbe dépendant directement de la couverture neigeuse, des températures et de la disponibilité en eau. Mais la diminution du manteau neigeux et la fusion nivale plus précoce au cours de la dernière décennie pourraient permettre à l'espace préalpin de développer des pratiques de pâturage hivernal.

Selon la Fédération des Alpages de l'Isère (ANEM, 2007), avec le réchauffement et l'absence de neige, les plantes puisent de façon anticipée dans les réserves hydriques du sol, cette utilisation étant préjudiciable au bon déroulement de la phase biologique estivale. A cette période, le stress hydrique représente un réel risque pour l'enherbement prairial, enjeu essentiel des estives. Avec un automne plus chaud et un hiver thermique plus tardif, la période d'activité biologique se prolonge même par une repousse herbacée tardive déjà observée en octobre. La mauvaise qualité nutritive de ce fourrage explique alors que les éleveurs constatent une diminution de la prise de poids moyenne des bovins, quelquefois de l'ordre de $50 \%$. La réduction prévisible de la productivité des prairies peut donc conduire progressivement à la réduction des élevages de ruminants mis à l'herbe en zones de moyenne montagne. Parallèlement, le décalage et le réchauffement saisonnier vont conditionner une recrudescence de 
maladies animales, ainsi que la remontée septentrionale et altitudinale de nouvelles espèces invasives (AFSSA, 2005).

Cependant, l'ensemble de ces évolutions, impacts et adaptations souffre encore de nombreuses incertitudes. Ainsi, d'un point de vue écosystémique, deux hypothèses principales coexistent lorsqu'on évoque le réchauffement climatique en montagne $: i$ ) une remontée totale de la distribution altitudinale des espèces végétales forestières va s'opérer, liée au postulat d'équilibre et à la conservation de la niche climatique ; ii) une remontée partielle intervient, liée à un déséquilibre et/ou à des phénomènes adaptatifs (Lenoir, 2009). En conséquence, la remontée générale de l'optimum altitudinal des espèces et le développement de communautés végétales plus thermophiles apporteront sûrement des réponses locales, dépendantes des massifs, qui déboucheront sur une vulnérabilité sociale et économique plus complexe que celle généralement présentée.

\section{Tourisme et pratiques hivernales}

Avec le plus grand domaine skiable équipé du monde (plus de 150 stations), un certain nombre d'impacts théoriques maintenant bien connus sur l'industrie du tourisme sont attendus en région Rhône-Alpes, surtout dans les Préalpes, suite à l'élévation des températures. Ainsi, le recul altitudinal de la limite nivale peut signifier le repli des pratiques sportives liées à la neige vers les stations de haute altitude. La réduction de l'hiver thermique $\left(<0^{\circ} \mathrm{C}\right)$ observée depuis 20 ans implique par ailleurs des modifications des habitudes touristiques intra-régionales (i.e. l'essentiel de la fréquentation actuelle des stations de moyennes montagnes) mais également internationales (choix prioritaires pour la haute montagne). Mais le réchauffement estival peut aussi devenir un atout pour le développement et la mise en valeur de nouvelles activités associées à une saison chaude plus longue. L'augmentation des précipitations printanières et/ou estivales pourrait cependant modifier certaines pratiques sportives ou touristiques. A l'inverse, la répétition d'épisodes secs et/ou caniculaires (sans parler de tendance pluviométrique qui n'est pas démontrée dans les Alpes), conjuguée à l'abandon de certaines pratiques agro-sylvo-pastorales, peuvent également nuire au maintien des paysages attractifs pour les touristes (alpages, forêts, espaces ouverts comme les pelouses alpines).

D'un point de vue statistique, et afin d'en assurer la rentabilité, un domaine de montagne peut être considéré comme une aire de ski potentielle si une couche de neige d'au moins $30 \mathrm{~cm}$ d'épaisseur subsiste 100 jours (entre le $1^{\text {er }}$ décembre et le 15 avril pour l'espace alpin français), au mois sept hivers sur dix. L'altitude est également une composante discriminante, particulièrement dans la durée de l'enneigement (5 à 6 mois à l'altitude moyenne Vercors-Chartreuse), mais aussi dans le nombre d'épisodes neigeux. La relation linéaire entre l'altitude et le nombre de jours de neige est explicite pour les altitudes supérieures à $800 \mathrm{~m}$, les stations iséroises situées à plus de $1200 \mathrm{~m}$ enregistrant par exemple entre 6 et 19 jours de neige en mars. Si l'on prend l'exemple de l'hiver 2006-2007, anormalement doux à l'échelle de toute la France (de 4 à $5^{\circ} \mathrm{C}$ audessus de la normale dans les Préalpes), les entrées atlantiques humides et douces ont favorisé un enneigement très faible dans le Vercors (moins de $50 \mathrm{~cm}$ ), notamment parce que la limite pluie-neige s'est située régulièrement au-dessus de $2000 \mathrm{~m}$ d'altitude (Bigot et al., 2009). La saison nivale a duré seulement de début janvier à début avril, lorsque la masse d'air la plus septentrionale s'est refroidie (la fin de la saison 
correspond aussi à un mois d'avril défini comme le plus chaud observé depuis 1900). L'enneigement de cet hiver est finalement l'un des plus faibles enregistrés depuis 1960 dans les Alpes (d'après le référentiel nivologique de Météo-France situé au Col de Porte). Cette forte variabilité intra- et interannuelle explique donc la grande vulnérabilité de l'exploitation économique fondée sur la ressource nivale. Cette fragilité est renforcée si l'on tient compte du fait que la ligne de neige, observée aujourd'hui en moyenne dans les Alpes françaises vers $1200-1500 \mathrm{~m}$ d'altitude, se déplacera sûrement vers $1800 \mathrm{~m}$, selon les prévisions des modèles numériques, obérant tous les projets.

Face aux variations climatiques enregistrées, les stratégies d'adaptation des stations de sports d'hiver peuvent être de deux types: les solutions techniques ou l'évolution plus drastique du secteur touristique (ANEM, 2007). Le changement des températures atmosphériques à la fin des années 1980 dans les Alpes a déjà entraîné le développement de l'enneigement artificiel et des technologies associées (enneigeurs, retenues collinaires, nouvelle gestion hydraulique, revégétalisation, ...). Mais la fabrication de neige de culture implique une grande disponibilité en eau, évaluée en moyenne à $4000 \mathrm{~m}^{3}$ pour l'enneigement d'un hectare. En dehors des éventuels conflits d'usage et conséquences environnementales (consommation et alimentation végétale, pollution, tarissement de la ressource), les limites climatiques pour la production de neige de culture ne sont déjà quasiment plus atteintes en moyenne montagne, à l'image de ce qui a été décrit précédemment dans le Vercors et la Chartreuse, avec le changement des fréquences thermiques pour les températures négatives et pour les températures minimales. Ainsi, les enneigeurs automatiques entrent généralement en fonction quand la température humide est de $-4{ }^{\circ} \mathrm{C}$, la meilleure qualité de neige (poudreuse) étant fabriquée lorsque la température de l'air est inférieure à $-6^{\circ} \mathrm{C}$ (Chaix, 2008). La conservation du manteau neigeux artificiel est alors très sensible aux périodes de redoux et à l'augmentation du nombre de jours hors-gel. L'enjeu est donc de pouvoir maintenir ces investissements déjà réalisés, ou bien d'envisager des adaptations complexes (partager les pertes via des assurances, changer d'activité pour aller vers une 'station quatre saisons', changer la localisation des activités, ...).

31 A cause des contrastes régionaux observés à l'échelle préalpine (entre les parties septentrionale, centrale et méridionale) dans les tendances pluviothermiques des dernières décennies, il faut aussi imaginer que les équilibres économiques basés sur la ressource nivale et touristique vont changer dans les prochaines années, certaines régions s'adaptant peut-être mieux que d'autres, de nouvelles sources d'attractivité apparaissant par ailleurs (sports d'été, mise en valeur du patrimoine culturel, ...). Le changement climatique est donc aussi un des facteurs de la prochaine compétitivité des régions de montagne entre-elles ou entre espaces touristiques. Finalement, quel que soit le type de station, les évolutions structurelles des destinations touristiques en montagne sont plutôt des trajectoires à long terme (Marcelpoil, 2008). Et, sans contester l'importance fondamentale de l'aléa climatique, la variabilité des goûts de la clientèle a un impact au moins aussi important sur ce secteur touristique que la variabilité du climat. 


\section{Conclusion}

Quoique difficile à étudier à cause du manque de longues séries stationnelles, l'analyse statistique sur la période 1959-2009 à l'échelle des massifs du Vercors et de la Chartreuse met en évidence plusieurs changements pluviothermiques significatifs. Le plus évident est la rupture de stationnarité postérieure à 1988, accompagnée d'une modification des fréquences thermiques (différente entre températures minimales et maximales) et d'un réchauffement à la fois hivernal et estival. Il semble également que ces changements puissent renforcer la typicité et le morcellement climatique régional dépendant de la topographie, compliquant l'étude de leur variabilité. Sans pouvoir le démontrer explicitement par modélisation écologique ou économique, ces variations climatiques intrasaisonnières et interannuelles constituent des mutations évidentes des principales contraintes environnementales régionales. Les transformations des phases bioclimatiques quotidiennes et des régimes ombrothermiques annuels vont interagir avec les cycles végétatifs, remodelant les activités agricoles et les paysages associés (ouverture des paysages, enfrichement, reforestation, biodiversité).

Les pistes d'adaptation sont actuellement nombreuses pour les domaines de moyenne montagne, passant notamment par le renforcement des observations météorologiques et hydrologiques, des systèmes de prévision et de modélisation du changement climatique, le développement de modèles socio-économiques dynamiques sur l'adaptation des individus et des sociétés, ou encore la diffusion de l'information à l'attention des acteurs des secteurs économiques concernés (Agence Européenne pour l'Environnement, 2009). Mais les adaptations sont actuellement mal connues et encore peu prévisibles, alors qu'elles peuvent sûrement permettre des économies d'échelle importantes pour des espaces sensibles comme la moyenne montagne. Une question plus large revient peut-être à mieux déterminer la fonction principale de l'espace montagnard dans un monde réchauffé, entre une réserve de biodiversité ou bien plutôt un lieu de production économique (MEEDDM, 2009).

\section{BIBLIOGRAPHIE}

AFSSA, 2005. Rapport annuel 2005 de l'Agence Française de Sécurité Sanitaire des Aliments. Consulté en septembre 2009 sur : http://www.afssa.fr/ , 108 p.

Agence Européenne pour l'Environnement, 2009. Regional climate change and adaptation. The Alps facing the challenge of changing water resources. EEA Technical report $\mathrm{N}^{\circ} 9$, European Environment Agency, Copenhague (Danemark), $148 \mathrm{p}$.

ANEM, 2007. Au-delà du changement climatique, les défis de l'avenir de la montagne. Rapport au 23 ème Congrès de l'Association Nationale des Elus de Montagne, 25 octobre 2007, 108 p.

Auer I., Böhm R., Jurkovic A., Orklik A., Potzmann R., Schöner W., Ungersböck M., Brunetti M., Nanni T., Maugeri M., Briffa K., Jones P., Efthymiadis D., Mestre O., Moisselin J.-M., Begert M., Brazdil R., Bochnice O., Cegnar T., Gajic-Capka M., Zaninovic K., Majstorovic Z., Szalai S., 
Szentimrey T. et Mercalli L., 2005. A new instrumental precipitation dataset for the greater alpine region for the period 1800-2002. Int. J. Climatol., n 25, p. 139-166.

Auer I., Böhm R., Jurkovic A., Lipa W., Orlik A., Potzmann R., Schöner W., Ungersböck M., Matulla C., Briffa K., Jones P. D., Efthymiadis D., Brunetti M., Nanni T., Maugeri M., Mercalli L., Mestre O., Moisselin J.-M., Begert M., Müller-Westermeier G., Kveton V., Bochnicek O., Stastny P., Lapin M., Szalai S., Szentimrey T., Cegnar T., Dolinar M., Gajic-Capka M., Zaninovic K., Majstorovic Z. et Nieplova E., 2007. HISTALP - Historical instrumental climatological surface time series of the greater Alpine region 1760-2003. Int. J. Climatol., n² 27, p. 17-46.

Bates B. C., Kundzewicz Z. W., Wu S. et Palutikof J. P. (éd.), 2008. Le changement climatique et l'eau. Document technique VI publié par le Groupe d'experts Intergouvernemental sur l'Evolution du Climat, Secrétariat du GIEC, Genève, 236 p.

Beniston M., Diaz H. F. et Bradley R. S., 1997. Climatic change at high elevation sites: an overview. Climatic Change, $\mathrm{n}^{\circ} 36$, p. 233-251.

Beniston M., 2006. Mountain weather and climate: a general overview and a focus on climatic change in the alps. Hydrobiologia, $n^{\circ} 562$, p. 3-16.

Bigot S., Laurent J.-P., Rome S. et Biron P.-E.,2006. Mise en place d'un observatoire pour le suivi écoclimatique des Hauts Plateaux du Vercors. Actes du XIX ${ }^{\mathrm{e}}$ colloque de l'Association Internationale de Climatologie, Epernay, p. 125-130.

Bigot S., Rome S., Planchon O. etLebel T.,2007. Variations climatiques et circulation atmosphérique européenne dans le massif du Vercors (1921-2006). Actes du XX ${ }^{\text {ème }}$ colloque de l'Association Internationale de Climatologie, Tunis, 111-116.

Bigot S. et Rome S.,2008. Etude des variations climatiques récentes dans les pré-Alpes françaises : le cas du Vercors. Bulletin de la Société Géographique de Liège, n 51, p. 63-77.

Bigot S., Rome S. et Dedieu J.-P., 2009. La variabilité interannuelle de l'enneigement dans les Préalpes françaises (massif du Vercors) : recherche de prédicteurs climatiques à l'échelle synoptique. Geographica Technica, numéro spécial, p. 73-78.

Casty C., Wanner H., Luterbacher J., Esper J. et Ohm R. B., 2005. Temperature and precipitation variability in the European Alps since 1500. Int. J. Climatol., n² 25, p. 1855-1880.

Chaix C., 2007. Climatologie hivernale des versants alpins (Savoie) : types de temps, températures et vents. Analyse des données météorologiques des domaines skiables. Thèse de doctorat de l'Université de Savoie, $306 \mathrm{p}$.

Durand Y., Laternser M., Giraud G., Etchevers P., Lesaffre B. et Mérindol L., 2009. Reanalysis of 44 years of climate in the French Alps (1958-2002): methodology, model validation, climatology and trends for air temperature and precipitation. Journal of Applied Meteorology and Climatology, $\mathrm{n}^{\circ} 48$, p. $429-449$

Frei C. et Schär C., 1998. A precipitation climatology of the Alps from high-resolution rain-gauge observations. Int. J. Climatol., n 18, p. 873-900.

Gautier D., 2004. Le pâturage hivernal dans les élevages ovins viande en zone préalpes. Etats des lieux, questions posées, enjeux et perspectives de développement. Compte rendu $\mathrm{N}^{\circ} 2043305$, coll. Résultats, Institut de l'Elevage, $103 \mathrm{p}$.

GIEC, 2007. Résumé à l'intention des décideurs. In 'Changements climatiques 2007 : Les éléments scientifiques. Contribution du Groupe de travail I au quatrième Rapport d'évaluation du Groupe d'experts Intergouvernemental sur l'évolution du Climat. [Solomon S., Qin D., Manning M., Chen 
Z., Marquis M., Averyt K.B., Tignor M. et Miller H.L. Eds, Cambridge University Press, Cambridge (RU), $18 \mathrm{p}$.

INRA, 2003. Le climat change, la nature et l'agriculture aussi! Quels impacts? Quelles adaptations? Février 2003, INRA, Mission communication.

Isoard S., Grothmann T. et Zebisch M., 2008. Climate change impacts, vulnerability and adaptation: Theory and concepts. Workshop 'Climate change impacts and adaptation in the European Alps: Focus water', UBA, Vienne (Autriche).

Lenoir J., 2009. Impacts d'un réchauffement rapide du climat sur la distribution des espèces végétales forestières le long du gradient d'altitude. Thèse de doctorat de l'Institut des Sciences et Industries du Vivant et de l'Environnement (Agro Paris Tech), 283 p.

Marcelpoil E., 2008. Changement climatique et territoires touristiques de montagne... De la question des impacts à celle des capacités de réponse. Dur'Alpes Attitude, article en ligne consulté en septembre 2009 : http://www.duralpes.com .

Matulla C., Schöner W., Alexandersson H., Von Storch H., Wang X. L., 2007. European storminess: late nineteenth century to present. Climate Dynamics, $n^{\circ} 31$, p. 125-130.

MEEDDM, 2009. Evaluation du coût des impacts du changement climatique et de l'adaptation en France. Rapport de la deuxième phase, septembre 2009, DGEC-ONERC, 554 p.

Moisselin J.-M., Schneider M., Canellas C. et Mestre O., 2002. Changements Climatiques en France au $20^{\mathrm{e}}$ siècle. Étude des longues séries de données homogénéisées françaises de précipitations et temperatures. La Météorologie, $\mathrm{n}^{\circ}$ 38, p. 45-56.

ONERC, 2008. Changement climatiques dans les Alpes : impacts et risques naturels. Rapport Technique $\mathrm{N}^{\circ} 1$ de l'ONERC, $100 \mathrm{p}$.

Ozenda P., 1985. La végétation de la chaîne alpine dans l'espace montagnard européen. Dunod, $330 \mathrm{p}$.

Rome S. et Bigot S., 2009. Les extrêmes thermiques dans le massif du Vercors (2004-2008). Geographica Technica, numéro spécial, p. 395-400.

Schmidli J., Schmutz C., Frei C., Wanner H. et Schär C., 2002. Mesoscale precipitation variability in the region of the European Alps during the $20^{\text {th }}$ century. Int. J. Climatol., $\mathrm{n}^{\circ} 22$, p. 1049-1074.

Spagnoli B., Planton S., Déqué M., Mestre O. et Moisselin J.-M., 2002. Detecting climate change at a regional scale, 2002: the case of France. Geophys. Res. Lett., n 29(10), p. 1450.

Terray L. et Braconnot P., 2007. Livre Blanc ESCRIME (Étude des Scénarios Climatiques). IPSL \& Météo-France, 39 p.

Trenberth K. E., Jones P. D., Ambenje P. G., Bojariu R., Easterling D. R., Klein Tank A. M. G., Parker D. E., Renwick J. A., Rahimzadeh F., Rusticucci M. M., Soden B. J. et Zhai P.-M., 2007. Observations: Surface and Atmospheric Climate Change. In Climate Change 2007. The Physical Science Basis. Contribution of WG 1 to the Fourth Assessment Report of the Intergovernmental Panel on Climate Change. [S. Solomon, D. Qin, M. Manning, Z. Chen, M. C. Marquis, K. B. Averyt, M. Tignor and H. L. Miller (eds)], Cambridge University Press, Cambridge (U. K.), p. 235-336.

Vidal J.-P., Martin E., Franchisteguy L., Baillon M. et Soubeyroux J.-M., 2009. A 50-year highresolution atmospheric reanalysis over France with the Safran system. Int. J. Climatol.,doi: 10.1002/joc.2003. 


\section{RÉSUMÉS}

Le climat des Alpes françaises reste encore mal décrit, et sa variabilité interannuelle est difficile à étudier du fait des spécificités topoclimatiques locales et du manque de séries climatiques longues. Les conséquences régionales du changement de certaines contraintes climatiques sur plusieurs secteurs d'activité sont pourtant redoutées. Cet article propose donc un diagnostic des variations climatiques contemporaines (1959-2009) à l'échelle des Préalpes du Nord (Vercors et Chartreuse) en utilisant des chroniques stationnelles pluviothermiques. Les changements observés sont ensuite mis en relation avec des impacts connus ou potentiels sur les activités socio-économiques régionales.

The climate of the French Alps remains poorly described, and its interannual variability is difficult to study because of the wide variety of local topoclimatic characteristics and the lack of available long-term time-series. Regional consequences by certain climatic threat on various economic activities are nevertheless expected. This article thus proposes a diagnosis of the contemporary climatic variations (1959-2009) on the scale of French northern Prealps (Vercors and Chartreuse) by using the time series of 4 reference stations. Observed changes are then related to known or potential impacts on the regional socioeconomic activities.

\section{INDEX}

Mots-clés : changement, climat, impacts, Préalpes, températures

Keywords : changes, climate, impacts, Prealps, temperatures

\section{AUTEURS}

\section{SYLVAIN BIGOT}

Directeur de la revue Climatologie, Sylvain Bigot est membre du Laboratoire LTHE (CNRS-IRDINPG, UMR 5564) et Professeur à l'université Joseph Fourier (Grenoble 1). sylvain.bigot@ujfgrenoble.fr

\section{SANDRA ROME}

Laboratoire LTHE (CNRS-IRD-INPG, UMR 5564) et Université Joseph Fourier (Grenoble 1). sandra.rome@ujf-grenoble.fr 